\title{
Research Article n-Gram-Based Text Compression
}

\author{
Vu H. Nguyen, ${ }^{1}$ Hien T. Nguyen, ${ }^{1}$ Hieu N. Duong, ${ }^{2}$ and Vaclav Snasel ${ }^{3}$ \\ ${ }^{1}$ Faculty of Information Technology, Ton Duc Thang University, Ho Chi Minh City, Vietnam \\ ${ }^{2}$ Faculty of Computer Science and Engineering, Ho Chi Minh City University of Technology, Ho Chi Minh City, Vietnam \\ ${ }^{3}$ Faculty of Electrical Engineering and Computer Science, VSB-Technical University of Ostrava, Ostrava, Czech Republic
}

Correspondence should be addressed to Hien T. Nguyen; hien@tdt.edu.vn

Received 21 May 2016; Revised 2 August 2016; Accepted 25 September 2016

Academic Editor: Geun S. Jo

Copyright (C) $2016 \mathrm{Vu} \mathrm{H}$. Nguyen et al. This is an open access article distributed under the Creative Commons Attribution License, which permits unrestricted use, distribution, and reproduction in any medium, provided the original work is properly cited.

\begin{abstract}
We propose an efficient method for compressing Vietnamese text using $n$-gram dictionaries. It has a significant compression ratio in comparison with those of state-of-the-art methods on the same dataset. Given a text, first, the proposed method splits it into $n$-grams and then encodes them based on $n$-gram dictionaries. In the encoding phase, we use a sliding window with a size that ranges from bigram to five grams to obtain the best encoding stream. Each $n$-gram is encoded by two to four bytes accordingly based on its corresponding $n$-gram dictionary. We collected $2.5 \mathrm{~GB}$ text corpus from some Vietnamese news agencies to build $n$ gram dictionaries from unigram to five grams and achieve dictionaries with a size of $12 \mathrm{~GB}$ in total. In order to evaluate our method, we collected a testing set of 10 different text files with different sizes. The experimental results indicate that our method achieves compression ratio around $90 \%$ and outperforms state-of-the-art methods.
\end{abstract}

\section{Introduction}

According to [1], data compression is a process of converting an input data stream into another data stream that has a smaller size. A stream can be a file, a buffer in memory, or individual bits sent on a communications channel. The main objectives of data compression are to reduce the size of input stream and increase the transfer rate as well as save storage space. Typically, data compression techniques are classified into two classes, that is, lossless and lossy, based on the result of the decompression phase.

Text compression is a field of data compression, which uses the lossless compression technique to convert an input file to another form of data file. It cannot use the lossy compression technique because it needs to recover the exact original file from the compressed file. If lossy compression technique was used, the meaning of the decompression file will be different from the original file. Several techniques have been proposed for text compression in recent years. Most of them are based on the same principle of removing or reducing redundancies from the original input text file. The redundancy can appear at character, syllable, or word levels. This principle proposed a mechanism for text compression by assigning short codes to common parts, that is, characters, syllables, words, or sentences, and long codes to rare parts.

In recent years, several techniques have been developed for text compression. These techniques can be further classified into four major types, that is, substitution, statistical, dictionary, and context-based method. The substitution text compression techniques replace a certain longer repetition of characters with a shorter one. A technique that is a representative of these techniques is run-length encoding [2]. The statistical techniques usually calculate the probability of characters to generate the shortest average code length, such as Shannon-Fano coding [3, 4], Huffman coding [5], and arithmetic coding $[6,7]$. The next type consists of dictionary techniques, which involve substitution of a substring of text by an index or a pointer code. They relate to a position in the dictionary of the substring. Representatives of these techniques are LZW [8], LZ77 [9], and LZ78 [10]. The last type is contextbased techniques, which involve the use of minimal prior assumptions about the statistics of the text. Normally, they use the context of the text being encoded and the history of the text to provide more efficient compression. Representatives of this type are Prediction by Partial Matching (PPM) [11] and Burrow-Wheeler transform (BWT) [12]. Every 
method has its own advantages and disadvantages when being applied in a specific field. None of the above methods has been able to achieve the best results in terms of compression ratio.

Normally, users will decide to choose the appropriate method based on their purposes. With systems that allow the reconstruction of information from the output not to be exactly the same as the input, we can use lossy methods, such as systems to compress images and voice messages. With systems that require the original data to be recovered exactly from the compressed data, we must use lossless methods, such as text compression systems.

This paper presents the first attempt at text compression using $n$-gram dictionaries, and the contribution has three attributes; that is, (1) it is a method for text compression using $n$-gram dictionaries, (2) it collects the text corpus of the Vietnamese language from the Internet and builds five $n$-gram dictionaries with nearly 500,000,000 n-grams, and (3) a test set of 10 different text files with different sizes to evaluate our new system and compare it with my two previous methods $[13,14]$ and also with other methods. The rest of this paper is organized as follows. Section 2 presents earlier work related to this effort. Section 3 presents our proposed method, and Section 4 presents our experiments and results. Our conclusions are presented in Section 5.

\section{Related Work}

In recent years, most text compression techniques have been based on dictionary, word, or character levels [15-18]. Reference [15] proposed a method to convert the characters in the source file to a binary code, where the most common characters in the file have the shortest binary codes and the least common have the longest. The binary codes are generated based on the estimated probability of the character within the file and are compressed using 8-bit character word length. In [16], the authors proposed a method that combined word with LZW. First, their method splits input text to word and nonword and then uses them as initial alphabet of LZW. Reference [17] proposed a technique to compress short text messages based on two phases. In the first phase, it converts the input text consisting of letters, numbers, spaces, and punctuation marks commonly used in English writing to a format which can be compressed in the second phase. In the second phase, it proposes a transformation which reduces the size of the message by a fixed fraction of its original size. In [18], the authors proposed a word-based compression variant based on the LZ77 algorithm and proposed and implemented various ways of sliding windows and various possibilities of output encoding. In a comparison with other word-based methods, their proposed method is the best. In these research, they do not consider the structure of words or morphemes in the text.

In addition, there are some approaches to text compression based on syllables, BWT. These approaches involve some languages that have morphology in the structure of words or morphemes (e.g., German, Arabic, Turkish, and Czech) such as in [19-23]. Reference [19] presented a new lossless text compression technique which utilizes syllable-based morphology of multisyllabic languages. The proposed method is designed to partition words into its syllables and then to produce their shorter bit representations for compression. The number of bits in coding syllables depends on the number of entries in the dictionary file. In [20], the authors proposed a genetic algorithm in syllable-based text compression. This algorithm was used to determine for the characteristic of syllables. These characteristics are stored into dictionary, which is part of the compression algorithm and it is not necessary to place them into compressed data. This leads to reduction of used space. In [21, 22], Lansky and his colleagues were the first to propose a method for syllable-based text compression techniques. In their papers, they focused on specification of syllables, methods for decomposition of words into syllables, and using syllable-based compression in combination of the principles of LZW and Huffman coding. In [23], the authors first proposed a method for small text file compression based on the Burrow-Wheeler transformation. This method combines the Burrow-Wheeler transform with the Boolean minimization at the same time.

In our previous papers for Vietnamese text compression $[13,14]$, we proposed a syllable-based method based on morphology and syllable dictionaries in [13]. With each morphosyllable, it is split into a consonant and a syllable, and they are compressed based on their corresponding dictionaries. This method has a compression ratio that converges to around $73 \%$, and it is suitable for small text files. The method in [14] compressed text based on a trigram model; it splits a text file into trigrams and compresses these trigrams using a trigrams dictionary. This method achieves an encouraging compression ratio around $83 \%$.

\section{Proposed Method}

In this section, we present a method for Vietnamese text compression using $n$-gram dictionaries. This model has two main modules. The first module is used for text compression and the second module performs decompression. Figure 1 describes our text compression model. In our model, we use $n$-gram dictionaries for both compression and decompression. We will describe the model in detail in the following subsections.

\section{1. n-Gram Theory and Dictionaries}

3.1.1. $n$-Gram Theory. In this paper, we use $n$-gram theory mentioned from [24]: an $n$-gram is a contiguous sequence of $n$ items from a given sequence of a text or speech. An item can be a phoneme, a syllable, a letter, a word, or a morphosyllable. In general, an item is considered as an atomic unit. An $n$ gram of one item, two items, or three items is referred to as a "unigram," a "bigram," or a "trigram," respectively. Larger sizes are sometimes referred to by the number of items $n$, for example, "four-gram" and "five-gram."

3.1.2. Dictionaries. Since we focus on Vietnamese, we build five different Vietnamese dictionaries of unigram, bigram, trigram, four grams, and five grams corresponding to the 


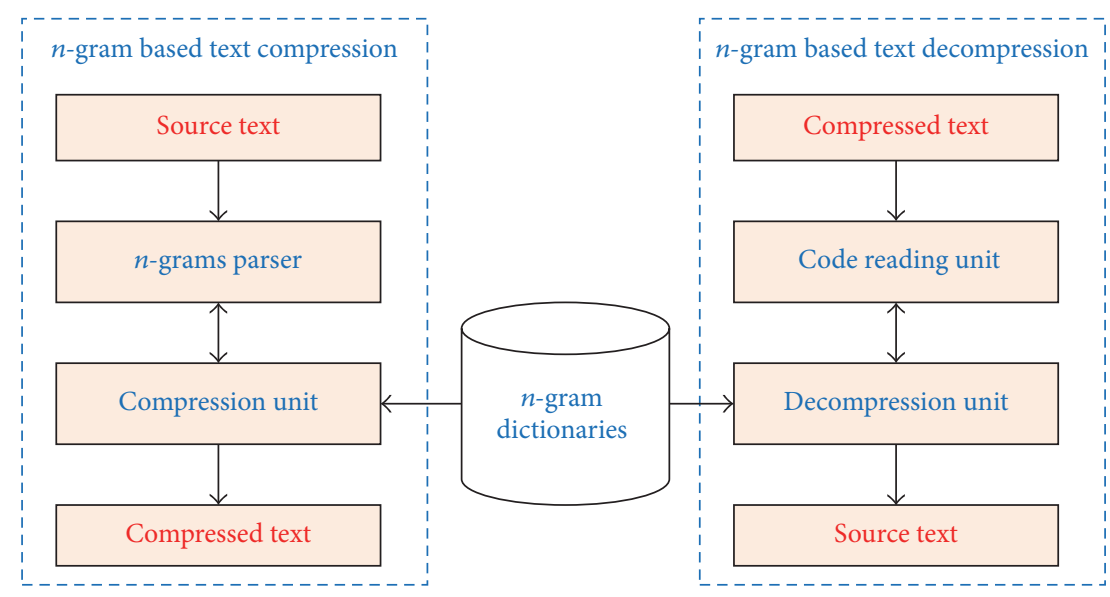

FIGURE 1: $n$-gram-based text compression model.

TABLE 1: $n$-gram dictionaries.

\begin{tabular}{lcc}
\hline$n$-gram dictionary & Number of $n$-grams & Size $(\mathrm{MB})$ \\
\hline 1 & 7,353 & 0.05 \\
2 & $20,498,455$ & 474 \\
3 & $84,003,322$ & 1,586 \\
4 & $169,916,000$ & 4,155 \\
5 & $225,203,959$ & 6,800 \\
\hline
\end{tabular}

number of grams compressed. Table 1 shows these dictionaries with their number of $n$-grams and size. These dictionaries have been built based on a text corpus collected from the Internet. The size of the text corpus is around $2.5 \mathrm{~GB}$. We use SRILM (http://www.speech.sri.com/projects/srilm/) to generate $n$-grams for these dictionaries. To increase the speed of searching in these dictionaries, we arranged them according to the alphabet. Table 1 describes the size and number of $n$-grams in each dictionary.

3.2. Compression. As presented in Figure 1, the compression module takes a source text as an input and then passes the text through two submodules, that is, $n$-grams parser and compression unit, to compress it. In following subsections, we explain in detail.

3.2.1. $n$-Gram Parser. $n$-gram parser has been used to read a source text file, splits it to sentences based on newline, and reads the number of grams in the combination with the result of the compression unit. In $n$-gram parser, we use five kinds of $n$-gram to store for unigram, bigram, trigram, four grams, and five grams. Based on the result of the compression unit, the $n$-gram parser decides how many grams will be read next. Algorithm 1 shows the pseudocode of this phase. If five grams was found in the five-gram dictionary, that is, index $>0$, the force_four_gram_compression function would be called to encode all previous $n$-grams (unigram, bigram, trigram, and four grams); then the compress function would be called to encode this five grams. Next, the $n$-gram parser reads next five grams in the input string. Otherwise, it would input: The source text file

output: The encoded stream

(1) inputstring = read source text file

(2) count $=$ number of grams in the inputstring

(3) while count $\geq 5$ do

(4) $\mathrm{st} 5=$ get first five grams of the inputstring

(5) index $=$ find(st5, five_gram_dict)

(6) if index $\geq 0$ then

(7) force_four_gram_compression(st4)

(8) outputstring $+=$ compress(index, 5)

(9) delete first five grams of the inputstring

(10) count $-=5$

(11) end

(12) else

(13) st $4+=$ get first gram of the inputstring

(14) delete first gram of the inputstring

(15) count $-=1$

(16) if number of grams of st $4=4$ then

(17) four_gram_compression(st4)

(18) end

(19) end

(20) end

(21) if count $>0$ then

(22) four_gram_compression(inputstring)

(23) end

Algorithm 1: Pseudocode of the compression phase.

split one leftmost gram of five grams for four grams and read one gram more from the input string for five grams. When the number of grams of four-gram was 4 , it calls the four gram_compression function.

Algorithm 2 shows the pseudocode of the four_gram compression function. This function is used to compress four grams if it occurs in four-gram dictionary. Otherwise, it splits one leftmost gram of the four-gram variable for the trigram variable. Similar to this function, we have the trigram_compression, the bigram_compression, and the unigram_compression function. 
input: The four-gram string, in this case is st 4

output: The encoded stream

(1) index $=$ find(st 4 , four_gram_dict)

(2) if index $\geq 0$ then

(3) force_trigram_compression(st3)

(4) outputstring $+=$ compress(index, 4)

(5) delete content of $s t 4$

(6) end

(7) else

(8) $\mathrm{st3}+=$ first gram of $s t 4$

(9) delete first gram of st4

(10) if number of grams of st $3=3$ then

(11) trigram_compression(st3)

(12) end

(13) end

Algorithm 2: Pseudocode of the four_gram_compression.

input: The four-gram string, in this case is st 4

output: The encoded stream

(1) while number of grams of st $4>0$ do

(2) $s t 3+=$ first gram of $s t 4$

(3) delete first gram of st4

(4) if number of grams of $s t 3=3$ then

(5) trigram_compression(st3)

(6) end

(7) end

(8) force_trigram_compression(st3)

Algorithm 3: Pseudocode of the force_four_gram_compression.

The force_four_gram_compression is called to encode all four-gram, trigram, bigram, and unigram when five-gram variable is found in the five-gram dictionary. Similar to this function, we have the force_trigram_compression, the force bigram_compression, and the force_unigram_compression function (Algorithm 3).

3.2.2. Compression Unit. The compression unit uses the result from the $n$-gram parser to decide how many grams will be compressed and what kind of $n$-gram dictionaries should be used. Based on the number of $n$-grams in each dictionary, we will construct the number of bytes to encode each $n$-gram corresponding to the dictionary. Table 2 describes the number of bytes used to encode each $n$-gram of each dictionary.

To classify the dictionary that was used to encode each $n$ gram and the other cases, we use three most significant bits (MSB) of the first byte of each encoded byte. Table 3 describes the value of these bits corresponding to each dictionary.

The index of each $n$-gram corresponding to each dictionary is encoded in the bits after the first three bits of the first byte. As seen in Table 3, there are two special cases for the $n$-gram dictionary: a newline and a unigram that does not appear in the unigram dictionary corresponding to a value of
TABLE 2: Number of encoded bytes for each $n$-gram of each dictionary.

\begin{tabular}{lcc}
\hline$n$-gram dictionary & Number of $n$-grams & Number of bytes \\
\hline 1 & 7,353 & 2 \\
2 & $20,498,455$ & 4 \\
3 & $84,003,322$ & 4 \\
4 & $169,916,000$ & 4 \\
5 & $225,203,959$ & 4 \\
\hline
\end{tabular}

TABLE 3: Value of three MSB and number of bytes.

\begin{tabular}{lcc}
\hline $\begin{array}{l}n \text {-gram } \\
\text { dictionary }\end{array}$ & Value of three MSB & $\begin{array}{c}\text { Number of bytes is read } \\
\text { more }\end{array}$ \\
\hline 1 & 0 & 01 \\
2 & 010 & 1 \\
3 & 011 & 3 \\
4 & 100 & 3 \\
5 & 101 & 3 \\
Newline & 110 & 0 \\
Others & 111 & $\begin{array}{c}\text { Value of five bits after } \\
\text { three first bits of current } \\
\text { byte }\end{array}$ \\
\hline
\end{tabular}

"newline" and "others." In these cases, the compression unit will encode as follows:

(i) When the result received from the $n$-gram parser is the newline, the compression unit will encode the value "110" for the first three bits of MSB, and the next five bits of this byte will have the value "00000."

(ii) When the result is the others, the three MSB of the first byte are "111" and the next five bits of this byte present the number of bytes which were used to encode this gram.

3.3. Decompression. As seen in Figure 1, the decompression module takes a compressed text as an input and then passes the text through two submodules, that is, code reading unit and decompression unit, to decompress it. We explain in detail in following subsections.

3.3.1. Code Reading Unit. First, this unit reads the compressed text from the compression phase. This result becomes the input sequence of the code reading unit. The code reading unit splits this input sequence byte to byte. Then, it reads the first byte of the input sequence and splits and analyzes the first three bits of this byte to classify the dictionary to which this $n$-gram belongs. Based on this result, this unit will read more bytes from the input sequence. Table 2 shows the number of bytes that the code reading unit reads after the first byte according to the classification of the dictionary. After reading these bytes, it transfers them to the decompression unit and repeats its work until the input sequence is null. 


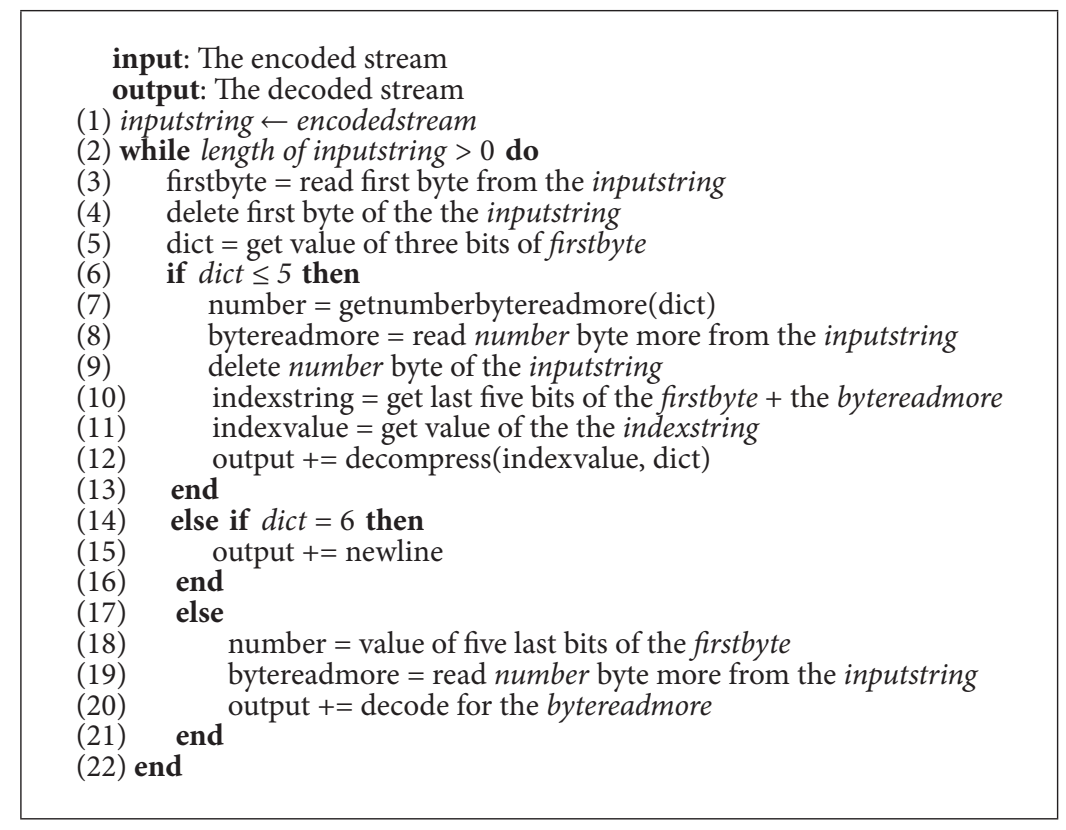

Algorithm 4: Pseudocode of the decompression phase.

3.3.2. Decompression Unit. This unit receives the results from the code reading unit. It decodes these results according to the classification of the dictionary as follows.

(i) Decode $n$-grams occurring in dictionaries

(1) Identifying the dictionary: based on the classification dictionary from the code reading unit

(2) Identifying the index of an $n$-gram in the dictionary: based on the value calculated from bytes that were read by the code reading unit

(3) Decode $n$-gram: when the classification of the dictionary has a value from one to five, the decompression unit decodes the $n$-gram in the dictionary based on the index of the $n$-gram

(ii) Decode $n$-grams that do not occur in dictionaries

(1) Decode newline: when the classification of dictionary is a "newline," it means that the value of the first three bits is 110 . The decompression unit decodes a newline for this $n$-gram

(2) Decode others: when the classification of the dictionary is "others," based on the value of the remaining bits of the first byte, the decompression unit will decode all bytes after the first byte

After finishing the decoding for one $n$-gram or other cases, the decompression unit reads the next result from the code reading unit and repeats the decompression tasks to decode other $n$-grams or other cases until it reads the last byte. Algorithm 4 shows the pseudocode of the decompression phase.

3.4. Compression Ratio. Compression ratio is used to measure the efficiency of the compression method. The stronger the compression ratio is, the better the quality of this method is. The compression ratio can be calculated by

$$
\mathrm{CR}=\left(1-\frac{\text { compressed_file_size }}{\text { original_file_size }}\right) \times 100,
$$

where original_file_size is size of the original file and compressed_file_size is size of the compressed file.

3.5. The Complexity of Our Method. Let $n$ be the number of $n$-grams in the source text and $a, b, c, d$, and $e$ be the number of five grams, four grams, trigrams, bigrams, and unigrams, respectively, in dictionaries. Let $k$ be $\log _{2}(a)+$ $\log _{2}(b)+\log _{2}(c)+\log _{2}(d)+\log _{2}(e)$. According to pseudocode from Algorithm 1, in the worst case, all five grams, four grams, trigrams, and bigrams do not occur in five grams, four grams, trigram, and bigram dictionary, respectively. Hence, the complexity of our method is $O(k * n)$.

\subsection{Example}

3.6.1. Compression Phase. Let us encode the following sequence using the $n$-gram approach.

Nén dũ liệu nhằm giảm kích thước dũ liệu dể tăng tốc độ truyền cũng nhu tiết kiệm không gian luiu trũ

Assume that we have five dictionaries for unigram, bigram, trigram, four grams, and five grams, as seen in Table 4.

The $n$-gram parser first encounters the first five-gram Nén dũ liệu nhằm giảm and copies it to the five-gram variable. This pattern is not in the five-gram dictionary, so it splits the first gram of this pattern for the four-gram variable and concatenates the next gram of the input sequence to the five-gram variable. The content of the five-gram and fourgram variables becomes dũ liệu nhằm giảm kích and Nén, 
TABLE 4: Five dictionaries.

(a) Unigram dictionary

\begin{tabular}{lc}
\hline Index & Entry \\
\hline 1 & nhằm \\
2 & lưu \\
3 & trữ \\
\hline
\end{tabular}

(b) Bigram dictionary

\begin{tabular}{lc}
\hline Index & Entry \\
\hline 1 & cũng như \\
\hline
\end{tabular}

(c) Trigram dictionary

\begin{tabular}{lc}
\hline Index & Entry \\
\hline 1 & Nén dũ̃ liệu \\
\hline
\end{tabular}

(d) Four-gram dictionary

\begin{tabular}{lc}
\hline Index & Entry \\
\hline 1 & tiết kiệm không gian \\
\hline
\end{tabular}

(e) Five-gram dictionary

\begin{tabular}{lc}
\hline Index & Entry \\
\hline 1 & giảm kích thước dữ liệu \\
2 & để tăng tốc độ truyền \\
\hline
\end{tabular}

respectively. Then, it checks the number of grams in the fourgram variable, which is one at this time. In this case, the value is less than four; it bypasses the four_gram_compression and turns back to the five-gram variable. Because this pattern is not in the five-gram dictionary, similar to the first case, it splits the first gram of this five-gram to the four-gram variable and concatenates the next gram of the input sequence to the five-gram variable. The content of the five-gram and fourgram variables shall become liệu nhằm giảm kích thước and Nén dü, respectively. Then, it checks the number of grams in the four-gram variable, which is two now. This value is less than four, similar to the first case; it turns back to five-gram variable. It repeats these operations until the content of the five-gram variable is nhằm giảm kích thước dũ and the fourgram variable is Nén dũ liệu. This five-gram pattern is not in five-gram dictionary, so it splits the first gram of this pattern for the four-gram variable and concatenates the next gram of the input sequence to the five-gram variable. The content of the five-gram and four-gram variables shall become giäm kich thước dũ liệu and Nén dũ liệu nhằm, respectively. It checks the number of grams in the four-gram variable, which is four now. It calls the four_gram_compression as presented in Algorithm 2. The four_gram_compression searches the fourgram pattern in the four-gram dictionary, which is not found in the four-gram dictionary. It splits the first gram of this pattern into the trigram variable. The content of the fourgram and the trigram variable becomes dũ liệu nhằm and Nén, respectively. Then, it checks the number of grams in the trigram variable, which is one at this time. So, it bypasses the trigram_compression, exits the four_gram_compression, and turns back to five-gram variable in Algorithm 1. The first five steps as seen in Table 5 show the content of the five-gram, four-gram, and trigram variables throughout these steps.

At Step 6, first, the $n$-gram parser checks the value of the five-gram variable in the five-gram dictionary. This pattern is in the dictionary; therefore, it calls the compression unit to encode all bigram, trigram, and four grams. Then, it encodes the five-gram. When the compression unit is finished, the $n$-gram parser reads the next five grams from the input sequence. In Table 5, Steps 6.1 to 6.4 show all substeps of Step 6 and in Table 6, Steps 6.2 to 6.4 show the encoder output sequence.

As seen in Table 5, at Step 6.1, the $n$-gram parser splits the first gram of the four-gram variable for the trigram variable, and the content of the four-gram and trigram variable shall become liệu nhămm and Nén dũ, respectively. Then, it checks the number of grams in the trigram variable, which is two at this time. So, it bypasses the trigram_compression and moves to Step 6.2. At Step 6.2, it continues splitting the first gram of the four-gram variable for the trigram variable. The content of the four-gram and trigram variables shall become nhằm and Nén dũ liệu, respectively. Next, it checks the number of grams in the trigram variable, which is three at this time. It then searches for this trigram in the trigram dictionary. Because this trigram is in the trigram dictionary, it calls the compression unit to encode bigram in the bigram variable. In this case, the bigram variable is null. It calls the compression unit to encode the trigram in the trigram variable and moves to the next substep. The encoded sequence of this trigram is shown in Table 6 at Step 6.2. The first three bits of this encoded sequence which have value 011 refer to trigram dictionary as seen in Table 3 and all remaining bits refer to the index of this trigram in the trigram dictionary.

At Step 6.3, the bigram and trigram variables are null; it counts the number of grams in the four-gram variable, which is 1 in this case; then it copies this gram to the unigram and searches for this unigram in the unigram dictionary. This unigram is in dictionary so it calls the compression unit to encode this unigram. The encoder output sequence of this unigram is shown in Table 6 at Step 6.3. At Step 6.4, it calls the compression unit to encode the five-gram in the five-gram variable, and the encoder output sequence of this five-gram is shown in Table 6 at Step 6.4. Then it reads the next five-gram in the input sequence to the five-gram variable. At this time, the content of the five-gram variable is để tăng tốc độ truyên.

The $n$-gram parser and the compression unit will process similar to previous cases for all remaining grams of the input sequence. The results of these steps are shown in Table 5 from Step 7 to Step 13.4. The encoder output sequences are shown in Table 6 from Step 7 to Step 13.4. The final encoder output sequence is the result of concatenation of all encoder output sequences from Step 6.1 to 13.4 in Table 6. The final encoder output sequence is

$01100000000000000000000000000001 \mid 00100000$
$00000001 \mid 10100000000000000000000000000001$
$|10100000000000000000000000000010| 01000000$
$000000000000000000000001 \mid 1000000000000000$
$0000000000000001|0010000000000010| 00100000$
00000011.

$01100000000000000000000000000001 \mid 00100000$ 00000001|10100000000000000000000000000001 |10100000000000000000000000000010|01000000 000000000000000000000001|1000000000000000 00000011. 
TABLE 5: All steps and values of $n$-grams.

\begin{tabular}{|c|c|c|c|c|}
\hline Step & Five-gram variable & Four-gram variable & Trigram variable & Bigram variable \\
\hline 1 & Nén dữ liệu nhằm giảm & & & \\
\hline 2 & dũ̃ liệu nhằm giảm kích & Nén & & \\
\hline 3 & liệu nhằm giảm kích thước & Nén dũ & & \\
\hline 4 & nhằm giảm kích thước dũ & Nén dũ liệu & & \\
\hline 5 & giảm kích thước dũ liệu & dũ liệu nhằm & Nén & \\
\hline 6.1 & giảm kích thước dũ̃ liệu & liệu nhằm & Nén dũ̃ & \\
\hline 6.2 & giảm kích thước dũ̃ liệu & nhằm & & \\
\hline 6.3 & giảm kích thước dũ liệu & & & \\
\hline 6.4 & để tăng tốc độ truyền & & & \\
\hline 7 & cũng như tiết kiệm không & & & \\
\hline 8 & như tiết kiệm không gian & cũng & & \\
\hline 9 & tiết kiệm không gian lưu & cũng như & & \\
\hline 10 & kiệm không gian lưu trũ & cũng như tiết & & \\
\hline 11 & không gian lưu trũ & như tiết kiệm & cũng & \\
\hline 12 & gian lưu trũ & tiết kiệm không & cũng như & \\
\hline 13.1 & lủu trũ̃ & tiết kiệm không gian & & \\
\hline 13.2 & & & & lưu trữ \\
\hline 13.3 & & & & trũ \\
\hline 13.4 & & & & \\
\hline
\end{tabular}

TABLE 6: Encoder output sequences.

\begin{tabular}{lcc}
\hline Step & Encoding of dictionary & Encoded sequence \\
\hline 6.2 & 011 & 00000000000000000000000000001 \\
6.3 & 001 & 0000000000001 \\
6.4 & 101 & 00000000000000000000000000001 \\
7 & 101 & 00000000000000000000000000010 \\
13.1 & 010 & 00000000000000000000000000001 \\
13.2 & 100 & 00000000000000000000000000001 \\
13.3 & 001 & 0000000000010 \\
13.4 & 001 & 0000000000011 \\
\hline
\end{tabular}

3.6.2. Decompression Phase. In this section, the encoder output sequence from the previous example is taken and is decoded using the decompression unit. The encoder output sequence in the previous example was

\section{$01100000000000000000000000000001 \mid 00100000$ 00000001|10100000000000000000000000000001 |10100000000000000000000000000010|01000000 000000000000000000000001|1000000000000000 0000000000000001|0010000000000010|00100000 00000011.}

The decompression unit uses the same dictionaries as the compression unit as seen in Table 4. It reads the first byte of the input sequence; the content of this first byte is 01100000 . The first three bits are split, and the value of these three bits is 011 . It finds the corresponding $n$-gram dictionary of these three bits and the number of bytes that is read more as presented in Table 3 . In this case, the $n$-gram dictionary is the trigram dictionary and the number of bytes that is read more is 3. The decoder reads the next three bytes from the input sequence. The index of the entry was calculated based on the value of all remaining bits after the first three bits and the three bytes that is read more. The entry is determined based on this index. The decoder repeats these steps until it reads the last byte of the input sequence. Table 7 shows all steps and results of the decompression phase.

The final decoder output sequence is the result of concatenation of all decoder output sequences from Step 1 to Step 8 as presented in Table 7. With each decoder output sequence from Step 1 to Step 7, we add one space character before the concatenation. The final encoder output sequence is Nén $d \tilde{u}$ liệu nhằm giảm kích thuiớc dũ liệu để tăng tốc độ truyên cũng nhu tiết kiệm không gian luiu trũ.

\section{Experiments}

We conducted an experiment to evaluate our method, using a dataset that is randomized collection from some Vietnamese news agencies. The dataset includes 10 files completely different in size and content.

In order to evaluate the effects of a combination of various $n$-gram dictionaries, we conducted three experiments with three kinds of systems. In the first case, we build a system with unigram, bigram, and trigram dictionaries. Next, we extend the first one with four-gram dictionary. Lastly, we extend the second one with five-gram dictionary. The results of the three experiments are shown in Table 8. As presented in Table 8, we find out that the compression ratio from the third case is 
TABLE 7: All steps and the results of the decompression phase.

\begin{tabular}{llllccc}
\hline Step & First byte & \multicolumn{3}{c}{ Dict. nbm bits to calculate index } & Index value & Decoder output sequence \\
\hline 1 & 01100000 & 011 & 3 & 00000000000000000000000000001 & 1 & Nén dữ liệu \\
2 & 00100000 & 001 & 1 & 0000000000001 & 1 & nhằm \\
3 & 10100000 & 101 & 3 & 00000000000000000000000000001 & 1 & giảm kích thước dũ̃ liệu \\
4 & 10100000 & 101 & 3 & 00000000000000000000000000010 & 2 & để tăng tốc độ truyền \\
5 & 01000000 & 010 & 3 & 00000000000000000000000000001 & 1 & cũng như \\
6 & 10000000 & 100 & 3 & 00000000000000000000000000001 & 2 & tiết kiệm không gian \\
7 & 00100000 & 001 & 1 & 0000000000010 & 3 & lưu \\
8 & 00100000 & 001 & 1 & 0000000000011 & trũ \\
\hline
\end{tabular}

TABLE 8: Compression ratio of three experience cases.

\begin{tabular}{lccccccc}
\hline Number & OFS & CFS-C1 & CR-C1 & CFS-C2 & CR-C2 & CFS-C3 & CR-C3 \\
\hline 1 & 1,166 & 210 & $81.99 \%$ & 166 & $85.76 \%$ & $88.34 \%$ \\
2 & 2,240 & 362 & $83.84 \%$ & 274 & $87.77 \%$ & 222 & $90.09 \%$ \\
3 & 6,628 & 1,245 & $81.22 \%$ & 999 & $84.93 \%$ & 887 & $86.62 \%$ \\
4 & 12,224 & 1,954 & $84.02 \%$ & 1,503 & $87.70 \%$ & 1,179 & $90.36 \%$ \\
5 & 22,692 & 3,565 & $84.29 \%$ & 2,652 & $88.31 \%$ & 2,180 & $90.39 \%$ \\
6 & 49,428 & 7,638 & $84.55 \%$ & 5,712 & $88.44 \%$ & 4,538 & $90.82 \%$ \\
7 & 96,994 & 15,636 & $83.88 \%$ & 12,359 & $87.26 \%$ & 10,416 & $89.26 \%$ \\
8 & 156,516 & 24,974 & $84.04 \%$ & 19,188 & $87.74 \%$ & 15,889 & $89.85 \%$ \\
9 & 269,000 & 43,887 & $83.69 \%$ & 34,182 & $87.29 \%$ & 28,937 & $89.24 \%$ \\
10 & 489,530 & 80,685 & $83.52 \%$ & 63,472 & $87.03 \%$ & 54,117 & $88.95 \%$ \\
\hline
\end{tabular}

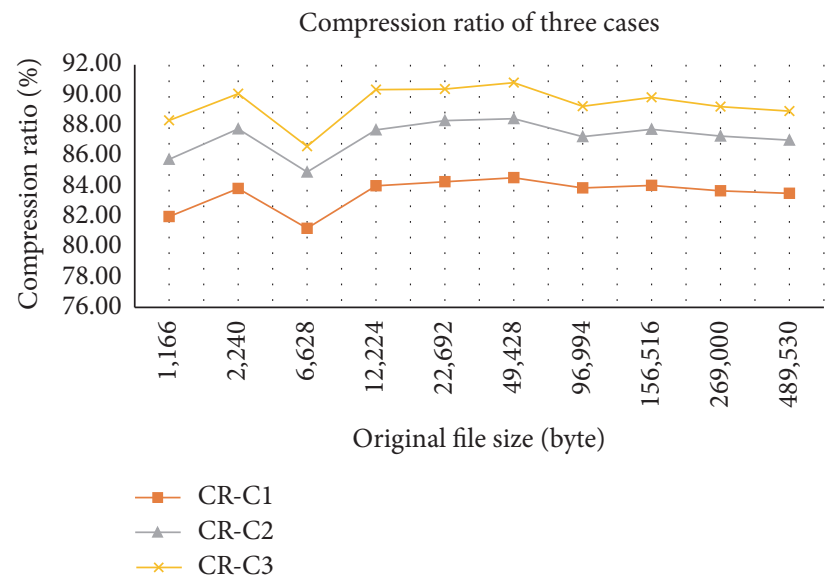

Figure 2: Comparison between the three cases.

the best, follow-up is the second case, and the last one comes from the first case. The compression ratio in this section was used according to (1). In Tables 8, 9, and 10 and Figures 2, $3,4,5$, and 6 , we have some abbreviations and meanings as follows: OFS: original file size in byte; CFS: compressed file size in byte; $\mathrm{CR}$ : compression ratio; $\mathrm{C} 1, \mathrm{C} 2$, and $\mathrm{C} 3$ : three cases above, respectively; O: our method; RAR: WinRAR; ZIP: WinZIP.

As seen in Figure 2, the compression ratio when we combine all five dictionaries is the highest.

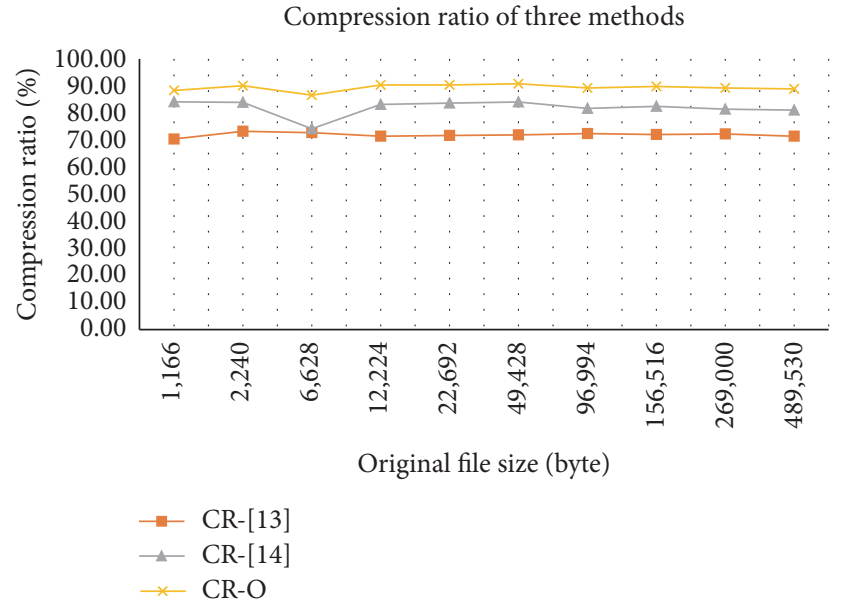

Figure 3: Compression ratio of our method $[13,14]$.

In order to evaluate our method with the methods presented in $[13,14]$, we compress the input files using these methods. In Table 9, we show the results of the current method in 10 cases in comparison with the methods in [13, 14]. As shown in Table 9 and Figure 3, the compression ratio of our method is better than the methods presented in $[13,14]$ for any size of text in our test cases.

Table 10 and Figure 4 show the results of our method in comparison with those of other methods, such as WinZIP version 19.5 (http://www.winzip.com/win/en/index.htm), the 
TABLE 9: CR of the current method with the methods presented in $[13,14]$.

\begin{tabular}{|c|c|c|c|c|c|c|c|}
\hline Number & OFS & CFS-[13] & CR-[13] & CFS-[14] & CR-[14] & CFS-O & CR-O \\
\hline 1 & 1,166 & 345 & $70.41 \%$ & 185 & $84.13 \%$ & 136 & $88.34 \%$ \\
\hline 2 & 2,240 & 599 & $73.26 \%$ & 359 & $83.97 \%$ & 222 & $90.09 \%$ \\
\hline 3 & 6,628 & 1,803 & $72.80 \%$ & 1,710 & $74.20 \%$ & 887 & $86.62 \%$ \\
\hline 4 & 12,224 & 3,495 & $71.41 \%$ & 2,057 & $83.17 \%$ & 1,179 & $90.36 \%$ \\
\hline 5 & 22,692 & 6,418 & $71.72 \%$ & 3,702 & $83.69 \%$ & 2,180 & $90.39 \%$ \\
\hline 6 & 49,428 & 13,881 & $71.92 \%$ & 7,870 & $84.08 \%$ & 4,538 & $90.82 \%$ \\
\hline 7 & 96,994 & 26,772 & $72.40 \%$ & 17,723 & $81.73 \%$ & 10,416 & $89.26 \%$ \\
\hline 8 & 156,516 & 43,701 & $72.08 \%$ & 27,434 & $82.47 \%$ & 15,889 & $89.85 \%$ \\
\hline 9 & 269,000 & 74,504 & $72.30 \%$ & 49,902 & $81.45 \%$ & 28,937 & $89.24 \%$ \\
\hline 10 & 489,530 & 139,985 & $71.40 \%$ & 92,739 & $81.06 \%$ & 54,117 & $88.95 \%$ \\
\hline
\end{tabular}

TABLE 10: Compression ratio of our method, WinRAR, and WinZIP.

\begin{tabular}{lccccccc}
\hline Number & OFS & CFS-O & CR-O & CFS-RAR & CR-RAR & CFS-ZIP & CR-ZIP \\
\hline 1 & 1,166 & 136 & $88.34 \%$ & 617 & $47.08 \%$ & 676 & $42.02 \%$ \\
2 & 2,240 & 222 & $90.09 \%$ & 887 & $60.40 \%$ & 946 & $57.77 \%$ \\
3 & 6,628 & 887 & $86.62 \%$ & 2,052 & $69.04 \%$ & 7111 & $68.15 \%$ \\
4 & 12,224 & 1,179 & $90.36 \%$ & 3,378 & $72.37 \%$ & 3,442 & $71.84 \%$ \\
5 & 22,692 & 2,180 & $90.39 \%$ & 6,162 & $72.85 \%$ & 6,150 & $72.90 \%$ \\
6 & 49,428 & 4,538 & $90.82 \%$ & 12,504 & $74.70 \%$ & 12,286 & $75.14 \%$ \\
7 & 96,994 & 10,416 & $89.26 \%$ & 21,389 & $77.95 \%$ & 21,321 & $78.02 \%$ \\
8 & 156,516 & 15,889 & $89.85 \%$ & 34,162 & $78.17 \%$ & 34,362 & $78.05 \%$ \\
9 & 269,000 & 28,937 & $89.24 \%$ & 56,152 & $79.13 \%$ & 57,671 & $78.56 \%$ \\
10 & 489,530 & 54,117 & $88.95 \%$ & 101,269 & $79.31 \%$ & & 108,175 \\
\hline
\end{tabular}

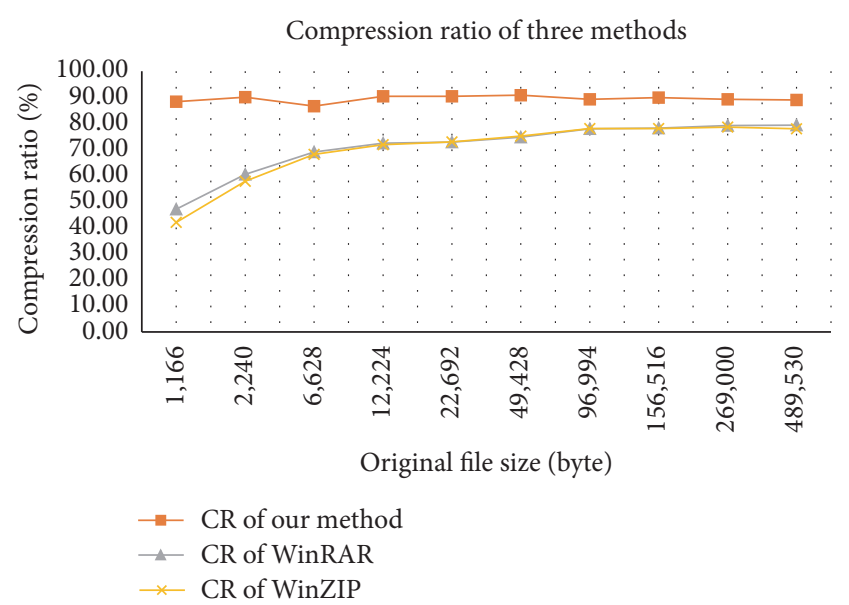

FIgURE 4: Compression ratio of our method, WinRAR, and WinZIP.

software combining LZ77 [8] and Huffman coding, and WinRAR version 5.21 (http://www.rarlab.com/download.htm), the software combining LZSS [25] and Prediction by Partial Matching [11]. The experimental results show that our method achieves the highest compression ratio on the same testing set.

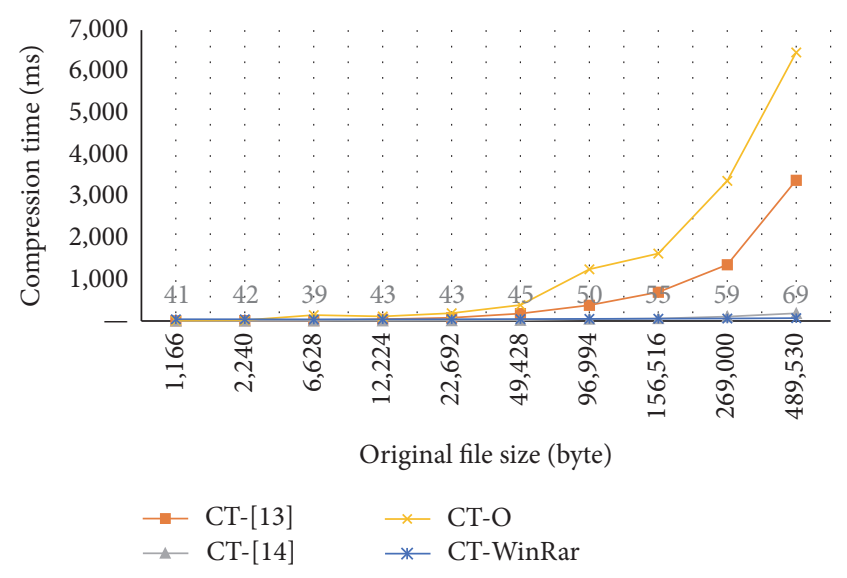

Figure 5: Compression time of four methods.

Tables 11 and 12 and Figures 5 and 6 show the compression and decompression time of our method in comparison with the methods in $[13,14]$ and WinRAR, respectively. In these tables and figures, we have some abbreviations and meanings as follows: CT: compression time; DT: decompression time; RAR: WinRAR; O: our method; ms: millisecond.

As presented in Table 12 and Figure 5, the compression time of our method is higher than those of other methods. 
TABLE 11: Compression time of four methods.

\begin{tabular}{lccccc}
\hline Number & File size & CT-[13]-ms & CT-[14]-ms & CT-O-ms & CT-RAR-ms \\
\hline 1 & 1,166 & 4 & 1 & 11 & 19 \\
2 & 2,240 & 8 & 2 & 143 & 42 \\
3 & 6,628 & 12 & 4 & 111 & 39 \\
4 & 12,224 & 43 & 5 & 187 & 43 \\
5 & 22,692 & 79 & 10 & 383 & 43 \\
6 & 49,428 & 181 & 41 & 1,246 & 55 \\
7 & 96,994 & 381 & 60 & 1,623 & 50 \\
8 & 156,516 & 692 & 105 & 3,374 & 59 \\
9 & 269,000 & 1,356 & 185 & 6,463 & 69 \\
\hline
\end{tabular}

TABLE 12: Decompression time of four methods.

\begin{tabular}{|c|c|c|c|c|c|}
\hline Number & File size & CT-[13]-ms & CT-[14]-ms & CT-O-ms & CT-RAR-ms \\
\hline 1 & 1,166 & 1 & 1 & 9 & 30 \\
\hline 2 & 2,240 & 2 & 2 & 17 & 32 \\
\hline 3 & 6,628 & 4 & 3 & 56 & 34 \\
\hline 4 & 12,224 & 8 & 8 & 83 & 37 \\
\hline 5 & 22,692 & 18 & 13 & 149 & 39 \\
\hline 6 & 49,428 & 70 & 18 & 329 & 42 \\
\hline 7 & 96,994 & 290 & 70 & 770 & 46 \\
\hline 8 & 156,516 & 961 & 250 & 1,398 & 54 \\
\hline 9 & 269,000 & 3,111 & 873 & 2,958 & 67 \\
\hline 10 & 489,530 & 11,427 & 5,070 & 6,773 & 98 \\
\hline
\end{tabular}

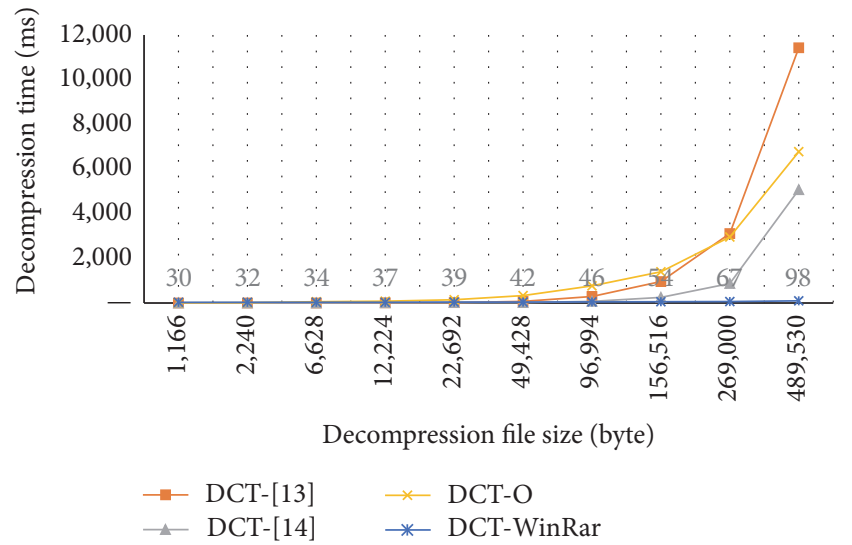

Figure 6: Decompression time of four methods.

As presented in Table 12 and Figure 6, the decompression time of our method is higher than [14] but it is slower than [13] and WinRAR.

\section{Conclusions}

In this paper, we present a novel method using $n$-gram dictionaries for text compression. We build five different $n$ gram dictionaries range from unigram to five grams from a $2.5 \mathrm{~GB}$ text corpus and obtain approximately $12 \mathrm{~GB} n$ grams. We conduct experiments on a dataset of 10 files with different sizes and content in three different scenarios. The first scenario uses unigram, bigram, and trigram dictionaries. The second scenario extends the first one with four-gram dictionary and the final scenario extends the second one with five-gram dictionary. The experimental results show that our method achieves the performance comparable with those of state-of-the-art methods including WinZIP and WinRAR in terms of compression ratio, while it is slower than these two of WinZIP and WinRAR. Speeding-up looking-up process of dictionaries may lead to foster the running time of ours method. We put this perspective as a direction of research in future.

\section{Competing Interests}

The authors declare that there is no conflict of interests regarding the publication of this paper.

\section{References}

[1] D. Salomon and G. Motta, Data Compression-The Complete Reference, Springer, New York, NY, USA, 5th edition, 2010.

[2] A. H. Robinson and C. Cherry, "Results of a prototype television bandwidth compression scheme," Proceedings of the IEEE, vol. 55, no. 3, pp. 356-364, 1967. 
[3] R. M. Fano, “The transmission of information," Tech. Rep., Massachusetts Institute of Technology, Research Laboratory of Electronics, Cambridge, Mass, USA, 1949.

[4] C. E. Shannon, "A mathematical theory of communication," The Bell System Technical Journal, vol. 27, no. 3, pp. 379-423, 1948.

[5] D. A. Huffman, "A method for the construction of minimumredundancy codes," Proceedings of the IRE, vol. 40, no. 9, pp. 1098-1101, 1952.

[6] P. G. Howard and J. S. Vitter, "Arithmetic coding for data compression," Proceedings of the IEEE, vol. 82, no. 6, pp. 857-865, 1994.

[7] I. H. Witten, R. M. Neal, and J. G. Cleary, "Arithmetic coding for data compression," Communications of the ACM, vol. 30, no. 6, pp. 520-540, 1987.

[8] T. A. Welch, "Technique for high-performance data compression," IEEE Computer, vol. 17, no. 6, pp. 8-19, 1984.

[9] J. Ziv and A. Lempel, "A universal algorithm for sequential data compression," IEEE Transactions on Information Theory, vol. 23, no. 3, pp. 337-343, 1977.

[10] J. Ziv and A. Lempel, "Compression of individual sequences via variable-rate coding," IEEE Transactions on Information Theory, vol. 24, no. 5, pp. 530-536, 1978.

[11] J. Cleary and I. Witten, "Data compression using adaptive coding and partial string matching," IEEE Transactions on Communications, vol. 32, no. 4, pp. 396-402, 1984.

[12] M. Burrows and D. Wheeler, "A block-sorting lossless data compression algorithm," Digital SRC Research Report, 1994.

[13] V. H. Nguyen, H. T. Nguyen, H. N. Duong, and V. Snasel, "A syllable-based method for vietnamese text compression," in Proceedings of the ACM 10th International Conference on Ubiquitous Information Management and Communication (IMCOM '16), $\mathrm{p}$. 17, Danang, Viet Nam, January 2016.

[14] V. H. Nguyen, H. T. Nguyen, H. N. Duong, and V. Snasel, "Trigram-based vietnamese text compression," in Recent Developments in Intelligent Information and Database Systems, vol. 642 of Studies in Computational Intelligence, pp. 297-307, Springer, 2016.

[15] H. Al-Bahadili and S. M. Hussain, "An adaptive character wordlength algorithm for data compression," Computers and Mathematics with Applications, vol. 55, no. 6, pp. 1250-1256, 2008.

[16] J. Dvorskp, J. Pokornp, and J. Snásel, “Word-based compression methods and indexing for text retrieval systems," in Proceedings of the 3rd East European Conference on Advances in Databases and Information Systems (ADBIS '99), pp. 75-84, Maribor, Slovenia, 1999.

[17] K. Kalajdzic, S. H. Ali, and A. Patel, "Rapid lossless compression of short text messages," Computer Standards \& Interfaces, vol. 37, pp. 53-59, 2015.

[18] J. Platos and J. Dvorskp, "Word-based text compression," CoRR abs/0804.3680, 2008.

[19] I. Akman, H. Bayindir, S. Ozleme, Z. Akin, and S. Misra, "A lossless text compression technique using syllable based morphology," The International Arab Journal of Information Technology, vol. 8, no. 1, pp. 66-74, 2011.

[20] T. Kuthan and J. Lansky, "Genetic algorithms in syllable-based text compression," in Proceedings of the Dateso Annual International Workshop on Databases, Texts, Specifications and Objects, Desna, Czech Republic, April 2007.

[21] J. Lansky and M. Zemlicka, “Text compression: syllables," in Proceedings of the Dateso Annual International Workshop on
Databases, Texts, Specifications and Objects, pp. 32-45, Desna, Czech Republic, April 2005.

[22] J. Lansky and M. Zemlicka, "Compression of small text files using syllables," in Proceedings of the Data Compression Conference, Snowbird, Utah, USA, March 2006.

[23] J. Platoš, V. Snášel, and E. El-Qawasmeh, "Compression of small text files," Advanced Engineering Informatics, vol. 22, no. 3, pp. 410-417, 2008.

[24] P. Koehn, Statistical Machine Translation, Cambridge University Press, Cambridge, UK, 2009.

[25] J. A. Storer and T. G. Szymanski, "Data compression via textual substitution," Journal of the ACM, vol. 29, no. 4, pp. 928-951, 1982. 

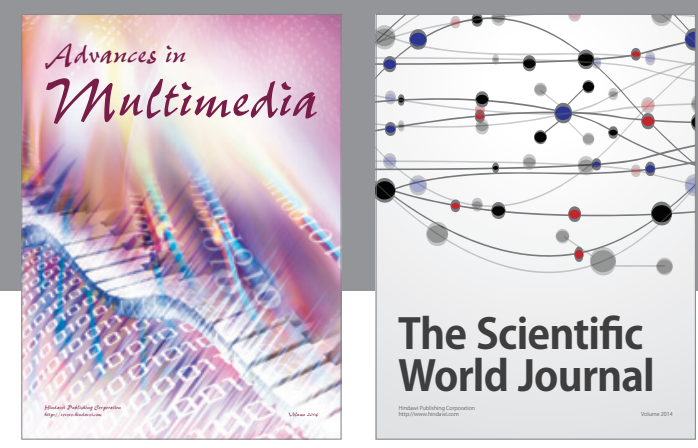

The Scientific World Journal
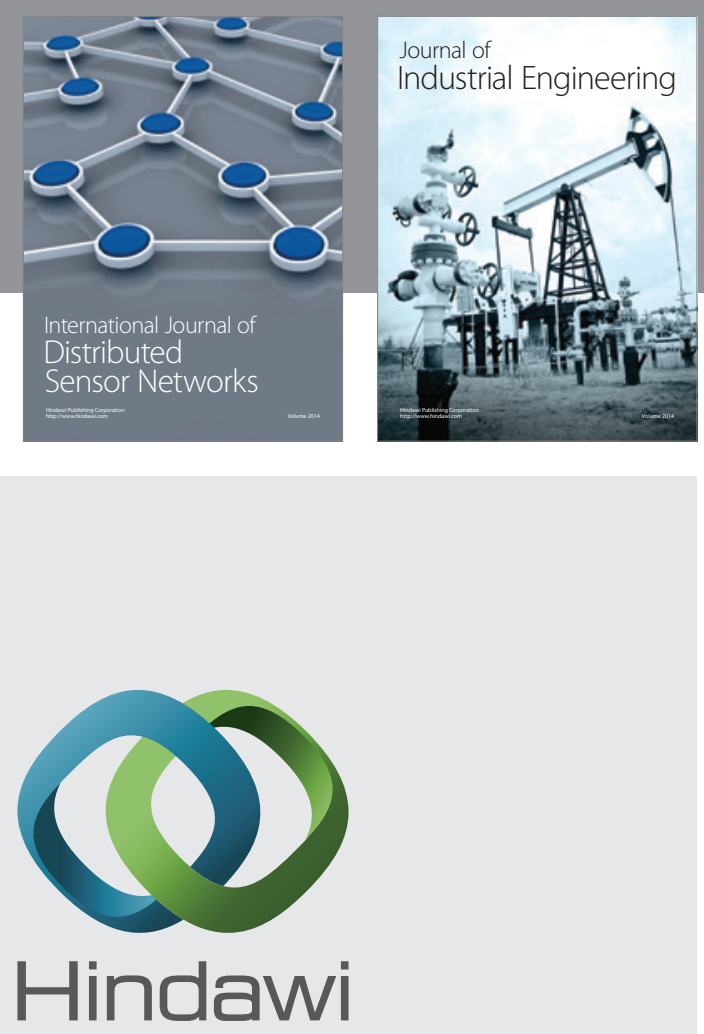

Submit your manuscripts at

http://www.hindawi.com

\section{Computer Networks} and Communications
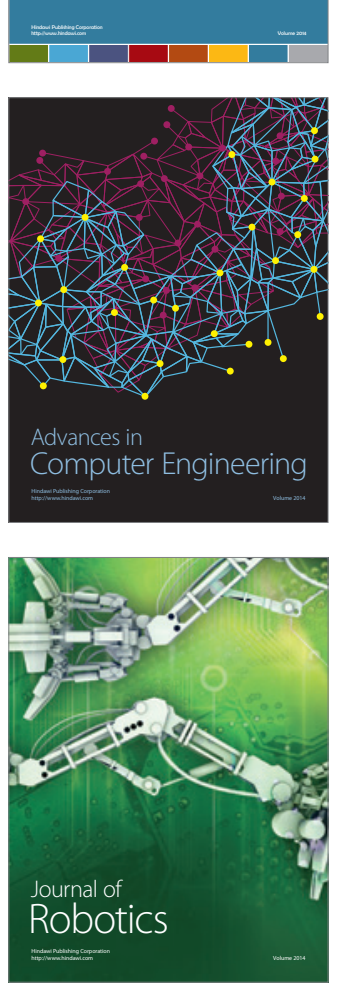
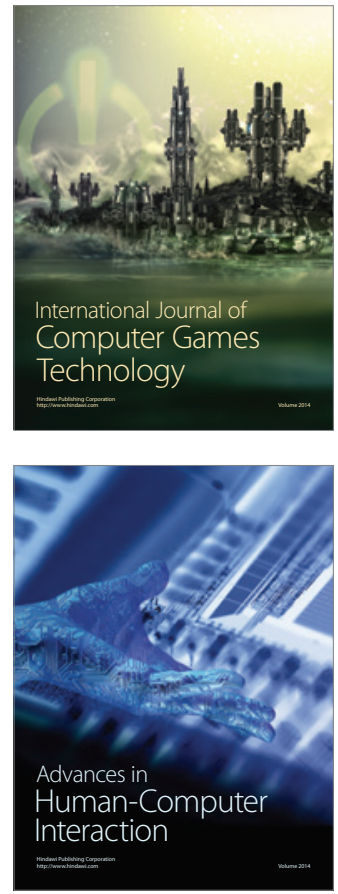
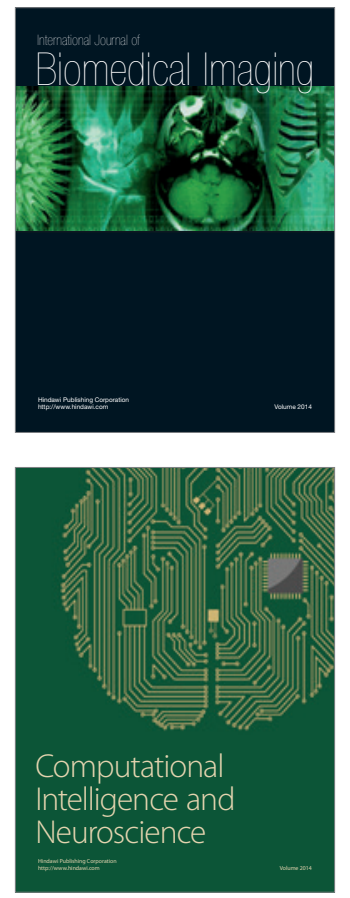
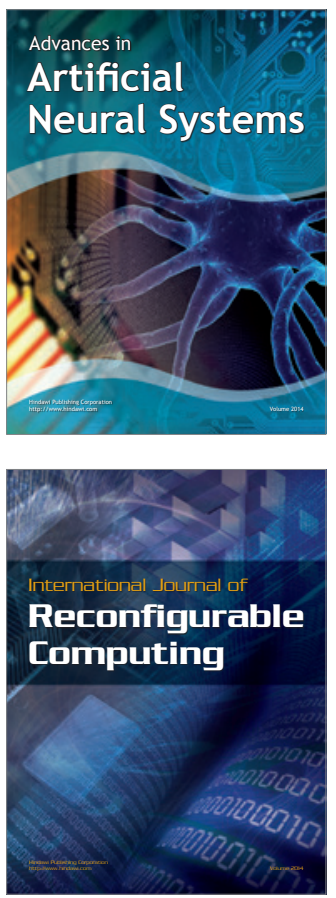
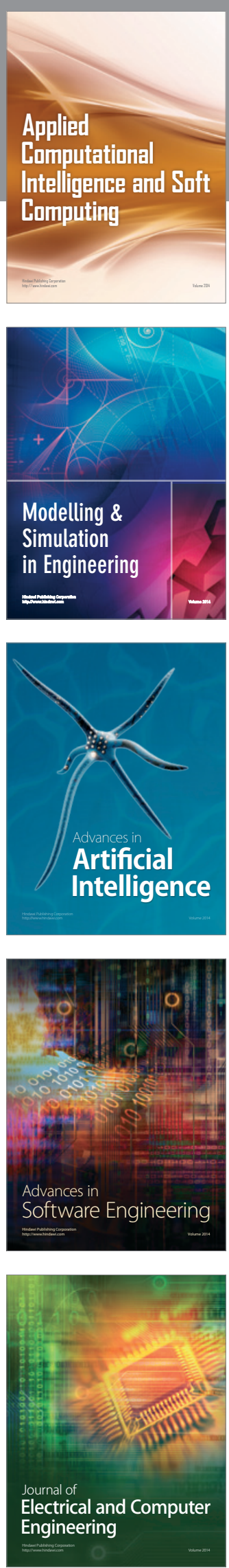